\title{
UNCOMPLEMENTED $C(X)$-SUBALGEBRAS OF $C(X)$ BY
}

\author{
JOHN WARREN BAKER
}

\begin{abstract}
In this paper, the uncomplemented subalgebras of the Banach algebra $C(X)$ which are isometrically and algebraically isomorphic to $C(X)$ are investigated. In particular, it is shown that if $X$ is a 0 -dimen sional compact metric space with its $\omega$ th topological derivative $X^{(\omega)}$ nonempty, then there is an uncomplemented subalgebra of $C(X)$ isometrically and algebraically isomorphic to $C(X)$.

For each ordinal $a \geq 1$, a class $\mathcal{C}_{a}$ of homeomorphic 0 -dimensional uncountable compact metric spaces is introduced. It is shown that each uncountable 0-dimensional compact metric space contains an open-and-closed subset which belongs to some $\mathcal{C}_{\alpha}$.
\end{abstract}

1. Introduction. Let $X$ and $Y$ be topological spaces and $\phi$ be a (continuous) map from $X$ onto $Y$. The induced linear operator $\phi^{0}$ is the multiplicative isometric isomorphism from $C(Y)$ into $C(X)$ that takes $f \in C(Y)$ into $f \phi$. A major result is

Theorem 4.6. If $X$ contains an open, 0-dimensional compact metric subspace $K$ with its $\omega$ th topological derivative $K^{(\omega)}$ nonempty, then there is a map $\phi$ of $X$ onto itself such that $\phi^{0}[C(X)]$ is uncomplemented in $C(X)$.

Observe that the hypothesis for $X$ is satisfied by all uncountable, 0-dimensional compact metric spaces (e.g., the Cantor set $\mathcal{C}$ ) and by the space $\Gamma(\alpha)$ of ordinals not exceeding $a$ provided $a \geq \omega^{\omega}$.

If $\phi$ is a map from $X$ onto $Y$ then an averaging operator for $\phi$ is a continuous linear operator $\mu$ from $C(X)$ into $C(Y)$ satisfying $\mu \phi^{0}(f)=f$ for $f \in C(Y)$. It is easy to see that $\phi$ admits an averaging operator for $\phi$ if and only if there is a projection $P$ of $C(X)$ onto its subalgebra $\phi^{0}[C(Y)]$ where $\mu$ and $P$ are related by $P=\phi^{0} \mu$ [21, Corollary 2.3]. As with most of the results of this paper, the conclusion to Theorem 4.6 can be stated in terms of averaging operators (i.e., there is a map of $X$ onto itself which does not admit an averaging operator).

The $\mathcal{C}_{a}$-spaces introduced in $\$ 4$ are formed by adding rays $\Gamma_{0}(\alpha)=\{\beta \mid \beta$ is an ordinal, $\beta<a\}$ to the Cantor set $\mathcal{C}$ so that each point in $\mathcal{C}_{a}$ is the limit of a

Received by the editors December 1, 1971.

AMS (MOS) subject classifications (1970). Primary 46B99, 46E15, 46J 10, 06A40; Secondary 02J05, 54G05, 54F05.

Key words and phrases. Banach spaces of continuous functions, Banach algebras of continuous functions, complemented subspaces of $C(X)$, averaging operators, compact 0-dimensional metric spaces, Boolean algebras.

Copyright $\odot 1974$, American Mathematical Society 
ray $\Gamma_{0}(a)$. A homeomorphic classification of $C_{a^{-s p a c e s ~ s i m i l a r ~ t o ~ t h e ~ c l a s s i c a l ~}}$ homeomorphic classification of the Cantor set is established (Lemma 4.3). The fact that each nondispersed 0 -dimensional compact metric space contains an openand-closed $\mathcal{C}_{a^{\text {sspace }}}$ for some $a$ provides a new technique for working with these spaces.

In the final section of this paper, two applications of the "uncomplemented $C(X)$-subalgebras of $C(X)$ " results are included. Theorem 5.1 and Theorem 5.2 are "uncomplemented" analogoues of A. Pel czyniski's "complemented $C(S)$-subspaces" theorems in his paper, On $C(S)$-subspaces of separable Banach spaces [22].

2. Preliminaries. The notation and terminology is that of Dunford and Schwartz's Linear operators. I [14] and Kelley's General topology [18] with the following exception: $A$ decomposition $D$ of a topological space $X$ is a disjoint collection of closed subsets of $X$ such that $X=\bigcup\{A: A \in D\}$ and the quotient space is denoted by $X / D$. An isomorphism $\mu$ between two Banach algebras which is multiplicative (i.e., $\mu(f g)=\mu(f) \mu(g)$ ) is called an algebra isomorphism.

For each subspace $S$ of $X$ let $S^{(1)}$ denote the set of all accumulation points of $S$ which are contained in $S$. Then $S^{(1)}$ is the complement in $S$ of the set of points which are isolated in the relative topology of $S$. If $\lambda$ is an ordinal, the topological derivative of order $\lambda$ of $S$, denoted $S^{(\lambda)}$, is defined by transfinite induction as follows: $S^{(0)}=S, S^{(\lambda)}=\left(S^{(a)}\right)^{(1)}$ if $\lambda=a+1$, and $S^{(\lambda)}=\bigcap_{a<\lambda} S^{(a)}$ if $\lambda$ is a limit ordinal.

The first limit ordinal is denoted by $\omega$ and the first uncountable ordinal by $\Omega$. We assume that all maps are continuous and that all topological spaces are Hausdorf $f$.

3. Construction of uncomplemented subalgebras. This section is closely related to the author's work in [4]. Most of the terminology and notations used in that paper are needed in this section and are used without being redefined.

If $D$ is a decomposition of a topological space $X$ and $q$ is the quotient map $D$, then $q^{0}$ is an isometric isomorphism from $C(X / D)$ onto the subalgebra of $C(X)$ consisting of the functions which are constant on each set in $D$. Frequently, this subalgebra of $C(X)$ is identified with $C(X / D)$ without specific reference to the isomorphism $q^{0}$. We follow Arens [3] and write $D=0$ when $D$ has no plural sets. The abbreviation u.s.c. is used for upper semicontinuous.

If $Z$ is a $D$-saturated subset of $X$, then the restriction of the decomposition $D$ to $Z$ is denoted $D_{Z}$. If $D$ is u.s.c., then $D_{Z}$ is u.s.c. and the identity map of $Z / D_{Z}$ onto $q(Z)$ is a homeomorphism (see [10, I, \$5.2, Proposition 4, p. 54]). If $Z$ is normal, then $Z / D_{Z}$ is normal $[15$, p. 85] and $q(Z)$ is a normal subset of $X / D$.

Lemma 3.1 and Proposition 3.2 are basically the same as Lemma 1.2 and Theorem 1.3, respectively, in [4]. The main difference is that the assumption that $X$ is normal is replaced by the assumption that a subspace $Z$ of $X$ is normal. 
The proofs of these two results are omitted, since their proofs are similar to the corresponding proofs in [4] and the only additional information needed is contained in the preceding paragraph. The purpose of Lemma 3.1 is to replace Lemma 1.2 of [4] in the proof of Proposition 3.2.

Lemma 3.1. Let $X$ be a topological space and let $D$ be an u.s.c. decomposition of $X$. Suppose there is a D-saturated, normal subspace $Z$ of $X$ and a plural set $Y$ of $D$ in Int $(Z)$ such that $D$ is contracting at $Y$ and the boundary $\partial Y$ of $Y$ contains at least $n$ points. If $P$ is a projection of $C(X)$ onto $C(X / D)$, then $\|P\| \geq 3-2 / n$.

Moreover, if $\epsilon>0$, if $U$ is a neighborbood of $Y$ and if $y_{1}, y_{2}, \cdots, y_{n}$ are distinct points in $\partial Y$, then there exist an $i$ and a neighborbood $V$ of $y_{i}$ such that for each $t$ in $V \sim Y$ there exists $f$ in $C(X)$ with $f \mid(X \sim U)=0,\|f\|=$ $f(t)=1$, and $P f(t)>3-2 / n-\epsilon$.

The following proposition establishes a lower bound for the norms of projections of $C(X)$ onto a $C(Y)$-subalgebra of $C(X)$. This proposition demonstrates that the existence of repeated limits of plural sets in the decomposition that $Y$ induces on $X$ can increase the norm of projections from $C(X)$ onto this $C(Y)$ subalgebra. This result substantially generalizes R. Arens's " $3-2 / n$ lower bound theorem" [3, Theorem 3.1] and extends a similar result obtained independently by S. Ditor to noncompact spaces [12, Corollary 5.4]. However, Ditor's result is more general in the compact case. The definition of $L_{n}\left(m_{1}, m_{2}, \cdots, m_{n}\right)$ is given in [4].

Proposition 3.2. Let $D$ be an u.s.c. decomposition of a topological space $X$ and $Z$ a normal subspace of $X$ such that Int $(Z)$ is D.saturated. If $D_{\operatorname{hr}(Z)}$ bas property $L_{n}\left(m_{1}, m_{2}, \cdots, m_{n}\right)$ and $P$ is a projection of $C(X)$ onto $C(X / D)$, then

$$
\|P\| \geq 2 n+1-\sum_{i=1}^{n} 2 / m_{i}
$$

Remarks 3.3. The "upper semicontinuous" requirement in Theorem 1.3 in [4] was inadvertently omitted.

Let $\phi$ be a map of a compact space $X$ onto a compact (Hausdorff) space $Y$ and let $\Delta_{\phi}$ be the decomposition $\left\{\phi^{-1}(y) \mid y \in Y\right\}$ of $X$. Then $\Delta_{\phi}$ is u.s.c., since $\phi$ is closed. It is interesting to observe that if $\Delta_{\phi}$ has property $L_{n}\left(m_{1}, m_{2}, \cdots, m_{n}\right)$, then using Ditor's definition in [12], $\Delta_{\phi}^{(n)}\left(m_{1}, m_{2}, \cdots, m_{n}\right)$ $\neq \varnothing$. Therefore, either by Proposition 3.2 or by Corollary 5.4 in [12], an averaging operator $U$ for $\phi$ has

$$
\|U\| \geq 2 n+1-\sum_{i=1}^{n} 2 / m_{i}=1+2 \sum_{i=1}^{n}\left(1-1 / m_{i}\right) .
$$


The next lemma is a more general form of the author's Lemma 2.4 in [4]. Both this lemma and the construction given in its proof are essential in the proofs of the theorems of this paper.

Lemma 3.3. (Construction of subspaces of $C(X)$ with high projection norm). Let $X$ be a topological space and $n$ a positive integer. Suppose $Z$ is a normal subspace of $X$ and $S$ is a subset of Int $(Z)$ with $S^{(n)} \neq \varnothing$ sucb that each point in $S^{(1)}$ bas a countable neighborbood base. Then for eacb positive integer $k>1$ there exists an u.s.c. decomposition $D$ of $X$ such that

(1) Each plural set in $D$ consists of $k$ elements of $S$.

(2) $X / D$ is Hausdorff. Moreover, $X / D$ is, respectively, normal, compact, first-countable, or compact and metrizable, provided $X$ bas the corresponding property.

(3) If $q$ is the quotient map from $X$ onto $X / D$, then $q^{0}$ is an algebraic isometric isomorphism from $C(X / D)$ into $C(X)$.

(4) The decomposition $D^{(j)}$ of $X$ contains a plural set if and only if $j<n$.

(5) If $P$ is a projection of $C(X)$ onto $C(X / D)$, then $\|P\| \geq 2 n-1-(2 n-2) / k$.

Proof. Let $x \in S^{(n)}$ and let $G$ be a closed neighborhood of $x$ included in Int $(Z)$. By induction, we select nonempty families $C_{1}, C_{2}, \ldots, C_{n+1}$ and $\mathcal{U}_{1}, \mathcal{U}_{2}, \ldots, \mathcal{U}_{n+1}$ of subsets of Int $(G)$ such that if $1 \leq m \leq n+1$ (and $C_{0}=\varnothing$ ) then

(a) $C_{1}$ consists of a singleton subset of $S$ and each set in $C_{m}$ for $m>1$ consists of $k$ elements from $S^{(n-m+1)}$.

(b) If $a \in A$ for some $A \in C_{m}$ and $U$ is a neighborhood of $a$, then $U$ includes a set in $C_{m-1}$ [i.e., $a$ is an accumulation point of sets in $C_{m-1}$ ].

(c) $\mathcal{U}_{m}$ is a family of disjoint, closed subsets such that for each $A$ in $C_{m^{\prime}}$ there is a neighborhood $U_{A}$ of $A$ in $\mathcal{U}_{m}$ which does not include any other set in $C_{m^{*}}$

(d) If $U \in \mathcal{U}_{m}$ then $U$ does not intersect any set in $C_{j}$ for $1 \leq j<m$.

(e) $\mathcal{U}_{m}$ implies $\mathcal{U}_{m-1}$ for $m>1$.

(f). The decomposition $D_{m}$ of $X$ consisting of the plural sets in $\left(\bigcup_{i=1}^{n-1} C_{i}\right) \cup \mathcal{U}_{m}$ is contracting and each set in $\mathcal{U}_{m}$ is a nonlimit set of $D_{m}$.

Let $C_{1}$ be the family consisting of the singleton set $\{x\}$ and let $\mathcal{U}_{1}=\{G\}$. It is easy to see that conditions (a)-(f) are satisfied for $m=1$.

Next, suppose $C_{1}, C_{2}, \ldots, C_{m}$ and $\mathcal{U}_{1}, \mathcal{U}_{2}, \ldots, \mathcal{U}_{m}$ have been selected and $m \leq n$. Let $A$ be a set in $C_{m}$. We may suppose $\bar{A}=\left\{a_{1}, a_{2}, \cdots, a_{z}\right\}$ where each $a_{i}$ is in $S^{(n-m+1)}$ where $z=1$ if $m=1$ and $z=k$ if $m>1$. There exists a neighborhood $U_{A}$ of $A$ in $\mathcal{U}_{m}$ which does not intersect any other set in $C_{m}$. There is a family $\left\{U_{i}\right\}_{i=1}^{z}$ of closed disjoint sets such that for each $i, U_{i}$ is a 
neighborhood of $a_{i}$ and is a subset of $U_{A}$. Let $\left\{V_{i j}\right\}_{j=1}^{\infty}$ be a closed monotone neighborhood base for $a_{i}$ with $V_{i j} \subset U_{i}$ for each $j$. In fact, we can suppose $\left\{V_{i j}\right\}_{j=1}^{\infty}$ is selected so that, for each $i$ and $j, V_{i j} \sim V_{i(j+1)}$ is a neighborhood of a point $a_{i j}$ in $s^{(n-m)}$. Then there is a family $\left\{W_{i j}\right\}_{j=1}^{\infty}$ of disjoint closed sets such that $W_{i j}$ is a neighborhood of $a_{i j}$ included in $V_{i j} \sim V_{i(j+1)}$. Then, we define

$$
\begin{aligned}
A_{i j} & =\left\{a_{i(j k+r)} \mid 1 \leq r \leq k\right\} \text { for } 1 \leq i \leq z, \\
U\left(A_{i j}\right) & =\bigcup_{r=1}^{k} W_{i(j k+r)} \text { for } 1 \leq i \leq z \text { and } j=0,1,2, \ldots, \\
C_{m+1} & =\left\{A_{i j} \mid A \in C_{m}, 1 \leq i \leq z \text {, and } j=0,1,2, \ldots\right\}, \\
U_{m+1} & =\left\{U\left(A_{i j}\right) \mid A \in C_{m}, 1 \leq i \leq z \text {, and } j=0,1,2, \ldots\right\} .
\end{aligned}
$$

Using these definitions, it is easy to see that hypotheses (a)-(e) are satisfied by $C_{m+1}$ and $U_{m+1}$. The proof that $(f)$ is satisfied is given in $[4$, paragraphs 2 and $3, \mathrm{p} .96]$. This completes the inductive selection of $C_{1}, C_{2}, \ldots, C_{n+1}$ and $u_{1}, u_{2}, \ldots, u_{n+1}$.

Let $D$ be the decomposition of $X$ consisting of the plural sets in $\bigcup_{j=1}^{n+1} C_{. j}$. It follows from Lemma 2.2 in [4] that since $D_{n+1}$ is contracting, $D$ is also contracting. (Let $M=D_{n+1}$ in Lemma 2.2.) This selection of $D$ is easily seen to satisfy conclusions (1) and (3). Let $q$ denote the quotient map of $D$. Since $D$ is u.s.c. and $G$ is a neighborhood of each plural set in $D, D_{G}$ (the restriction of $D$ to $G$ ) is u.s.c. and the identity map of $G / D_{G}$ onto $q(Z)$ is a homeomorphism $\left[10, I, \$ 5.2\right.$, Proposition 4, p. 53]. Since $G$ is normal, $G / D_{G}$ is normal and $q(G)$ is a normal subspace of $X / D$. A similar argument with $G$ replaced by $Z$ shows $q(Z)$ is a normal subspace of $X / D$. But $q(G) \subset q($ Int $Z)=$ Int $q(Z)$, and $q$ is a homeomorphism on $X \sim G$. Since $X \sim G$ is Hausdorff, it follows that $X / D$ is Hausdorff. If $X$ is normal or compact, then $X / D$ has the corresponding property [15, pp. 85 and 104]. If $X$ is both compact and metrizable, it follows by a theorem of K. Morita and S. Hanai [19, Theorem 1] and also by A. H. Stone [26, Theorem 1] that $X / D$ is also compact and metrizable. If $X$ is first countable, $X / D$ is also first countable since each decomposition set contains at most $k$ elements. Thus $D$ satisfies conclusion (2) also.

The following generalization of $(5)$ is established next:

$\left(5^{\prime}\right)$ If $M$ is an u.s.c. decomposition of $X$ such that $M$ contains each plural set of $D$ and $M$ is contracting at each plural set in $D$, then each projection $P$ of $C(X)$ onto $C(X / M)$ has $\|P\| \geq 2 n-1-(2 n-2) / k_{\text {. }}$

To establish $\left(5^{\prime}\right)$, we let $S_{i}=D^{(i)} \sim D^{(i+1)}$ for $i=1,2, \ldots, n-1$. Since each set in $S_{i}$ is a plural set in $D$ and $M$ is contracting at each of these sets, $M$ satisfies property $L_{n-1}(k, k, \cdots, k)$. By Proposition 3.2, each projection 
$P$ of $C(X)$ onto $C(X / M)$ has $\|P\| \geq 2 n-1-(2 n-2) / k$. This proves (5'). Letting $D=M$, we obtain (S).

To establish (4), observe that it follows by induction that the decomposition $D^{(m)}$ satisfies the following properties provided $0 \leq m \leq n$ :

(i) $\bigcup_{j=2}^{n-m+1} C_{j}$ is the family of plural sets in $D^{(m)}$. $D^{(m)}$.

(ii) The family of plural sets in $C_{n-m+1}$ is the set of nonlimit plural sets in

By (i), $D^{(m)}$ contains a plural set if and only if $n-m+1 \geq 2$ or $m \leq n-1$. This proves (4).

In case the collection $S^{(n)}$ in Lemma 3.3 contains an isolated point with respect to its subset topology, the decomposition $D$ of Lemma 3.3 can be selected so that two additional properties are satisfied.

Lemma 3.4. If $S^{(n)} \sim S^{(n+1)}$ is nonempty in Lemma 3.3, then the decomposition $D$ can be also selected so that

$\left(^{\prime}\right)$ Each plural set of $D^{(j)} \sim D^{(j+1)}$ consists of $k$ elements of $S^{(j)} \sim S^{(j+1)}$.

(6) For eacb ordinal number $a, t \in S^{(a)}$ if and only if $q(t) \in q(S)^{(a)}$.

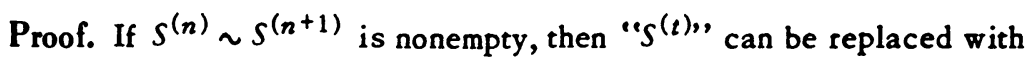

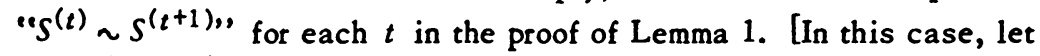
$x \in\left(S^{(n)} \sim S^{(n+1)}\right)$.] By the revised form of inductive hypothesis (a), it follows that each plural set in $C_{n-m+1}$ consists of $k$-points from $S^{(m)} \sim S^{(m+1)}$. This establishes ( $\left.1^{\prime}\right)$.

Next, we establish (6) by transfinite induction. It is obvious if $a=0$. Suppose $(6)$ is valid for all $a<\gamma$ where $1<\gamma$. Let $x \in s^{(\gamma)}$. Then for each $a<\gamma$, there exists a sequence $\left\{x_{n}\right\}$ of distinct points in $S^{(a)}$ such that $x_{n} \rightarrow x_{0}$. By inductive hypothesis, $\left\{q\left(x_{n}\right)\right\} \subset q(S)^{(a)}$ and since $q\left(x_{n}\right) \rightarrow$ $q(x), q(x) \epsilon^{n} q(S)^{(y)}$.

Conversely, let $q(x) \in q(S)^{(\gamma)}$. Then, for each $a<\gamma$, there exists a sequence $\left\{y_{n}\right\}$ of distinct points in $q(S)^{(a)}$ such that $y_{p} \rightarrow q(x)$. Choose $x_{n} \in S$ with $q\left(x_{n}\right)=y_{n}$. By inductive hypothesis, $x_{n} \in S^{(a)}$. If $q(x)$ is a singleton set, then $x_{n} \rightarrow x$ and it follows that $x \in S^{(\gamma)}$. If $x$ is a plural set, then $\gamma<\omega$ by $\left(1^{\prime}\right)$ and $x^{n}=\left\{z_{1}, z_{2}, \ldots, z_{k}\right\}$ for some choice of $z_{i}$ in $S$. Let $U_{1}, U_{2}, \cdots, U_{k}$ be disjoint neighborhoods of $z_{1}, z_{2}, \cdots, z_{k}$, respectively. Since $D$ is contracting, we may assume each $U_{i}$ is $(D \sim\{q(x)\})$-saturated. As before, there is a sequence $\left\{x_{n}\right\}$ of distinct points in $s^{(\gamma-1)}$ such that $q\left(x_{n}\right) \rightarrow q(x)$. But an infinite subsequence $\left\{x_{n_{i}}\right\}$ of $\left\{x_{n}\right\}$ is included in some $U_{j}$. Then $x_{n_{i}} \rightarrow z_{j}$, so $z_{j} \in S^{(\gamma)}$. But by (1'), $z_{j} \in S^{(\gamma)} \stackrel{n}{\text { implies }}\left\{z_{1}, z_{2}, \cdots, z_{k}\right\} \subset S^{(\gamma)}$. Since $x \in q(x), x \in S^{(y)}$.

Lemmas 3.5 and 3.6 are, respectively, the uncomplemented analogues of

Lemmas 3.3 and 3.4 . 
Lemma 3.5. (Construction of uncomplemented subspaces of $C(X))$ ). Suppose $Z$ is a normal subspace of a topological space $X$ and $S$ is a subset of Int ( $Z$ ) with $S^{(\omega)} \neq \varnothing$ such that each point in $S^{(1)}$ bas a countable neigbborbood base. Then for each positive integer $k>1$ there exists an u.s.c. decomposition sucb that conclusions (1)-(3) of Lemma 3.3 are satisfied. Moreover,

(4) The decomposition $D^{(j)}$ of $X$ contains a plural set if and only if $j<\omega$.

(5) The subspace $C(X / D)$ of $C(X)$ is uncomplemented in $C(X)$.

Proof. Suppose $x \in S^{(\omega)}$. Let $\left\{O_{n}\right\}_{n=1}^{\infty}$ be an open monotone neighborhood base for $x$ with $O_{1} \subset$ Int $(Z)$. We may assume this neighborhood base is selected so that $O_{n} \sim O_{n+1}$ is a neighborhood of a point $x_{n} \in S^{(n)}$. Let $E_{n}$ be a closed neighborhood of $x_{n}$ included in $O_{n} \sim O_{n+1}$. If $R$ is the decomposition of $X$ consisting of the plural sets $\left\{E_{n}\right\}_{n=1}^{\infty}$, then $R$ is clearly contracting. Let $S_{n}=S \cap$ Int $\left(E_{n}\right)$. Since $x_{n} \in S_{n}$, it follows by $\left(5^{\prime}\right)$ of Lemma 3.3 that there is a contracting decomposition $M_{n}$ of $X$ with each plural set in $M_{n}$ a subset of $S_{n}$ such that if $M$ is a contracting decomposition containing each plural set in $M_{n}$ and $P$ is a projection of $C(X)$ onto $C(X / M)$, then $\|P\| \geq n$. Moreover, each $M_{n}$ can be selected so that each plural set contains exactly $k$ points of $S_{n^{*}}$ Let $D$ be the decomposition consisting of the plural sets in $M_{n}$. By Lemma 2.2 in [4], $D$ is contracting. Thus, there does not exist a projection $P$ of $C(X)$ onto $C(X / D)$, since $\|P\| \geq n$ for each positive integer $n$ is impossible. This proves (5). Parts (1) and (3) are trivial, and the proof of (2) is the same as in Lemma 3.3. Part (4) follows, since the decomposition $M_{n}$ has the property that $M_{n}^{(j)}$ contains a plural set if and only if $j<n$.

Lemma 3.6. If $S^{(\omega)} \sim S^{(\omega+1)}$ is nonempty in Lemma 3.5, then the decomposition $D$ can also be selected so that

(1') Each plural set of $D^{(j)} \sim D^{(j+1)}$ consists of $k$ elements of $S^{(j)} \sim S^{(j+1)}$.

(6) For eacb ordinal number $a, t \in S^{(a)}$ if and only if $q(t) \in q(S)^{(a)}$.

Proof. If $S^{(\omega)} \sim S^{(\omega+1)}$ is nonempty, then the point $x$ in the proof of Lemma 3.5 can be selected from $S^{(\omega)} \sim S^{(\omega+1)}$. Also, the points $x_{n}$ in that proof can be selected from $S^{(n)} \sim S^{(n+1)}$. Then by Lemma 3.4, each of the decompositions $M_{n}$ in the proof of Lemma 3.5 can be selected so that $M_{n}$ satisfies $\left(1^{\prime}\right)$. Since each plural set of $D$ is contained in some $M_{n},\left(1^{\prime}\right)$ is established.

The proof of (6) is identical to the proof of (6) in Lemma 3.4 .

4. Existence of uncomplemented $C(X)$-subalgebras of $C(X)$. In this section, wo apply the lemmas of the last section to certain topological spaces $X$ to construct a subalgebra of $C(X)$ isometrically and algebraically isomorphic to $C(X)$ that either has a large lower bound for the norms of projections from $C(X)$ or is uncomplemented in $C(X)$. 
A topological space $X$ is dispersed (scattered) if it does not contain a perfect subset. If $X$ is dispersed, there is a least ordinal a such that $X^{(a)}$ is either finite or empty. The characteristic (characteristic system) of $X$ is the ordered pair $(\alpha, n)$ where $n$ is the cardinality of $X$. Note that $n \geq 1$ if $X$ is compact. If $\xi$ is an ordinal, $\Gamma(\xi)$ denotes the space of ordinals not exceeding $\xi$ with the interval topology and $\Gamma_{0}(\xi)$ denotes the subspace of $\Gamma(\xi)$ consisting of the ordinals strictly less that $\xi$.

Theorem 4.1. Let $n$ be a positive integer. Suppose a topological space $X$ includes a compact first-countable set $K$ sucb that Int $(K)^{(n)}$ contains infinitely many isolated points. Then for each $\epsilon>0$, there is a map $\phi$ of $X$ onto itself such that if $P$ is a projection of $C(X)$ onto $\phi^{0}[C(X)]$, then $\|P\| \geq 2 n+1-6$.

Proof. Let $x$ be an accumulation point of the set of isolated points in Int $(K)^{(n)}$. Suppose $\left\{U_{j}\right\}_{j=1}^{\infty}$ is a neighborhood base for $x$. Let $\left\{x_{j}\right\}_{j=1}^{\infty}$ be a sequence of distinct isolated points of Int $(K)^{(n)}$ with $x_{j}$ in $U_{j}$. By induction, there is a sequence $\left\{V_{j}\right\}_{j=1}^{\infty}$ of disjoint closed sets such that $V_{j}$ is a neighborhood of $x_{j}$ included in Int $(K) \cap U_{j}$. Since $X^{(n+1)}$ is closed and $X^{(n)} \sim X^{(n+1)}$ is discrete in its subset topology, we may assume $V_{j} \cap X^{(n)}=\left\{x_{j}\right\}$. Each $v_{i}$ is dispersed and compact; hence, it is 0-dimensional [20]. Thus, there exists an openand-closed (in $V_{j}$ ) neighborhood $W_{j}$ of $x_{j}$ included in Int $\left(V_{j}\right)$. Since $W_{j} \subset$ Int $\left(V_{j}\right), W_{j}$ is open-and-closed in $X$. Let $W=\bigcup_{i=1}^{\infty} W_{i}$ and $S=W \cup\{x\}$. The set $S$ is first-countable, compact and has characteristic $(n+1,1)$, so it follows by a theorem due to $Z$. Semadeni [24] (see [5, Corollary 2]) that $S$ is homeomorphic to $\Gamma\left(\omega^{n+1}\right)$.

Let $\epsilon>0$ and choose a positive integer $k$ sufficiently large so that $(2 n) / k<6$. Since $S^{(n+1)} \neq \varnothing$, it follows by Lemma 3.3 that there is an u.s.c. decomposition $D$ of $X$ such that each projection $P$ of $C(X)$ onto $C(X / D)$ has $\|P\| \geq 2 n+1$ $(2 n) / k>2 n+1-\epsilon$. Since $S^{(n+1)} \sim S^{(n+2)}=\{x\}$, it follows by Lemma 3.4 that $D$ can also be selected so that if $q$ denotes the quotient map, then $x \in S^{(a)}$ if and only if $q(x) \in q(S)^{(a)}$ for each ordinal $a$. Thus, $q(S)$ also has characteristic $(n+1,1)$. The subset $q(S)$ is compact because $S$ is compact. But $q(S)$ is firstcountable as each plural set of $D$ is finite, so, by [5, Corollary 2], $q(S)$ is also home omorphic to $\Gamma\left(\omega^{n+1}\right)$.

Let $\mu$ be a homeomorphism of $S$ onto $q(S)$. As topological derivatives are preserved by homeomorphisms [23, Lemma 3.1 $(e)],\{\mu(x)\}=\mu\left(S^{(n+1)}\right)=\mu(S)^{(n+1)}=$ $q(S)^{(n+1)}=\{q(x)\}$ and $\mu(x)=q(x)$. Since $x$ is the only possible accumulation point of $X \sim S$ contained in $S$, we can extend $\mu$ to a map of $X$ into $q(X)$ by letting $\mu(x)=q(x)$ for $x$ in $X \sim S$. Then $\mu$ is one-to-one and onto $X / D$. Since $q(x)$ is a singleton set and $q^{-1}$ is continuous on $q(X) \sim q(S), \mu$ is a homeomorphism of $X$ onto $X / D$. 
Thus, $\phi=\mu^{-1} \circ q$ is a map of $X$ onto $X$ and $\phi^{0}[C(X)]=q^{0}[C(X / D)]$. Consequently, each projection $P$ of $C(X)$ onto $\phi^{0}[C(X)]$ has $\|P\| \geq 2 n+1-6$.

Our first "uncomplemented $C(X)$-algebra of $C(X)$ " result is contained in the next theorem. This theorem is essentially the uncomplemented version of Theorem 4.1.

Theorem 4.2. Suppose a topological space $X$ includes a first-countable compact subset $K$ such that Int $(K)^{(n)}$ contains an isolated point for each positive integer n. Then there is a map $\phi$ of $X$ onto itself sucb that $\phi^{0}[C(X)]$ is an uncomplemented subalgebra of $C(X)$.

Proof. Let $t_{n}$ be an isolated point in Int $(K)^{(n)}$ for each positive integer $n$. Let $x$ be an accumulation point of $\left\{t_{n}\right\}_{n=1}^{\infty}$. Suppose $\left\{U_{n}\right\}$ is a neighborhood base

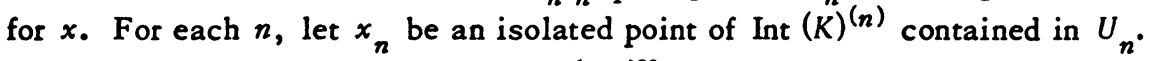
By induction, there exists a sequence $\left\{V_{n}\right\}_{n=1}^{\infty}$ of disjoint sets such that $V_{n}^{n}$ is a neighborhood of $x_{n}$ included in Int $(K) \cap U_{n}$. Since $X^{(n+1)}$ is closed in its subset topology, we may assume $V_{n} \cap X^{(n)}=\left\{x_{n}\right\}$ for each $n$.

The remainder of the proof follows the proof of Theorem 4.1, except that $n+1$ is replaced with $\omega$ and Lemmas 3.5 and 3.6 are used in place of Lemmas 3.3 and 3.4 respectively.

Next, for each denumerable ordinal number $a$, we construct a compact subspace $\mathcal{C}_{a}$ of the unit interval satisfying the two following properties: (1) $\left(\mathcal{C}_{a}\right)^{(a)}$ $=\mathcal{C}$, and (2) each point in the subset $\mathcal{C}$ of $\mathcal{C}_{a}$ is the limit of a well-ordered sequence in $\mathcal{C}_{a} \sim \mathcal{C}$ homeomorphic to $\Gamma_{0}\left(\omega^{\alpha}\right)$. Let $I_{n, k}$ denote the $k$ th open interval removed (counting from left to right) in the $n$th step of the construction of the Cantor set $\mathcal{C}[17, \mathrm{p} .70]$. Let $a_{n, k}$ and $b_{n, k}$ denote the left and right endpoint, respectively, of $I_{n, k}$ and let $m_{n, k}$ be the midpoint of $I_{n, k}$. In each interval $\left[a_{n, k} m_{n, k}\right.$ ) select a well-ordered sequence $A_{n, k}=\left\{a_{\mu}\right\}_{\mu<\omega} a$ homeomorphic to $\Gamma_{0}(\omega)$ with $\lim _{\mu<\omega a} a_{\mu}=a_{n, k}$. Similarly, select a well-ordered sequence $B_{n, k}=\left\{b_{\mu}\right\}_{\mu<\omega^{a}}$ in $\left.C_{n, k^{,}} b_{n, k}^{n, k}\right]$ homeomorphic to $\Gamma_{0}\left(\omega^{a}\right)$ with $\lim _{\mu<\omega^{a}} b_{\mu}=b_{n, k}$. Then let $\mathcal{C}_{a}^{\mu}$ be the subspace of $[0,1]$ defined by

$$
\mathcal{C}_{a}=\mathcal{C} \cup\left[\bigcup_{n, k=1}^{\infty}\left(A_{n, k} \cup B_{n, k}\right)\right] \text {. }
$$

Clearly, the choice of each $A_{n, k}$ and $B_{n, k}$ is possible but not unique. The fact that $\mathcal{C}_{a}$ is independent up to homeomorphism of the choices of $A_{n, k}$ and $B_{n, k}$ is established in Proposition 4.4. It follows from Lemma 1 in [5] and the construction of $\mathcal{C}_{a}$ that $\mathcal{C}_{a}$ satisfies properties (1) and (2) of the preceding paragraph. Compact metric spaces which satisfy (1) and (2) will be called $\mathcal{C}_{a^{\text {-spaces. }} \text {. }}$ More specifically, 
Definition. Let $\alpha$ be an ordinal number. A topological space $X$ is called a $C_{a^{-}}$ space if and only if $X$ is an uncountable 0-dimensional compact metric space, $X^{(a)}$ is perfect, and each point in $X^{(a)}$ is the limit of a well-ordered sequence in $X \sim X^{(a)}$ homeomorphic to $\Gamma_{0}\left(\omega^{a}\right)$.

If $X$ is a topological space, there is a least ordinal $\lambda$ such that $X^{(\lambda)}=$ $X^{(\lambda+1)}$. Let $\operatorname{Ker}(X)=X^{(\lambda)}$ and observe that $\operatorname{Ker}(X)$ is the largest perfect subset of $X$.

An alternate characterization of $\mathcal{C}_{a^{-s p a c e s}}$ is given by the following lemma.

Lemma 4.3. Let $X$ be a compact 0-dimensional metric space. If $a$ is an ordinal number, then $X$ is a $\mathcal{C}_{a^{-s p a c e}}$ if and only if

$$
\operatorname{Ker}(X) \subset \mathrm{Cl}\left[X^{(\gamma)} \sim \operatorname{Ker}(x)\right]
$$

for eacb $\gamma<a$, but $X^{(a)}=\operatorname{Ker}(X) \notin \varnothing$.

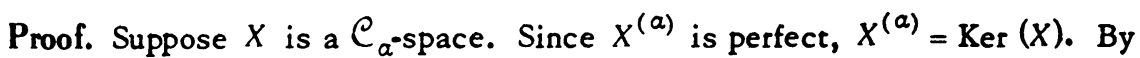
Lemma 1 in [5] it follows that $\operatorname{Ker}(X) \subset \mathrm{Cl}\left[X^{(\gamma)} \sim \operatorname{Ker}(X)\right]$ for each $\gamma<a$.

Conversely, suppose $\operatorname{Ker}(X) \subset \mathrm{Cl}\left[X^{(\gamma)} \sim \operatorname{Ker}(X)\right]$ for each $\gamma<a$, but $X^{(a)}=\operatorname{Ker}(X) \neq \varnothing$. Thus, $X^{(a)}$ is perfect. The proof that each point in $X^{(a)}$ is the limit of a well-ordered sequence of points in $X \sim X^{(a)}$ homeomorphic to $\Gamma_{0}\left(\omega^{a}\right)$ is similar to the proof of Theorem 2 in [7] and is omitted.

Each uncountable, 0-dimensional, perfect, compact metric space is homeomor-

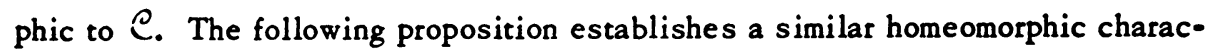
terization for each $\mathcal{C}_{a}$.

Proposition 4.4. All $\mathcal{C}_{a^{-s p a c e s ~ a r e ~ b o m e o m o r p h i c . ~}}$

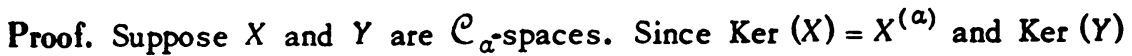
$=Y^{(a)}$ are nonempty, 0-dimensional, perfect metric spaces, they are both homeomorphic to the Cantor set. Thus, there is a homeomorphism $\phi$ of $\operatorname{Ker}(X)$ onto $\operatorname{Ker}(Y)$. Let $G_{1}=X \sim \operatorname{Ker}(X)$ and $G_{2}=Y \sim \operatorname{Ker}(Y)$. Since $G_{1}^{(\gamma)}$ and $G_{2}^{(\gamma)}$ are infinite for each $\gamma<\alpha$ and $G_{1}^{(a)}=G_{2}^{(a)}=\varnothing$, it follows by Theorem 3 in [5] that both $G_{1}$ and $G_{2}$ are homeomorphic to $\Gamma_{0}\left(\omega^{a}\right)$. By Lemma 4.3, $\phi\left[\operatorname{Ker}(X) \cap \mathrm{Cl}\left[X^{(\gamma)} \sim \operatorname{Ker}(X)\right]\right]=\phi(\operatorname{Ker} X)=\operatorname{Ker}(\gamma)=\operatorname{Ker}(\gamma) \cap \mathrm{Cl}\left[Y^{(\gamma)} \sim \operatorname{Ker}(Y)\right]$ for each $\gamma<a$. Since $\mathrm{Cl}\left[X^{(\gamma)} \sim \operatorname{Ker}(X)\right]=\mathrm{Cl}\left[Y^{(\gamma)} \sim \operatorname{Ker}(X)\right]=\varnothing$ for all $\gamma \geq \alpha$, it follows that $\phi\left(\operatorname{Ker}(X) \cap \mathrm{Cl}\left[X^{(\gamma)} \sim \operatorname{Ker}(X)\right]\right)=\operatorname{Ker}(Y) \cap \mathrm{Cl}\left[Y^{(\gamma)} \sim \operatorname{Ker}(Y)\right]$ for each ordinal number $\gamma$. By Theorem 1.1 in [23], $\phi$ can be extended to a homeomorphism of $X$ onto $Y$.

It is well known that a nondispersed compact metric space $X$ contains a subset $K$ homeomorphic to the Cantor set. However, consideration of the case $X=\mathcal{C}_{1}$ indicates that even when $X$ is 0 -dimensional, it is sometimes impossible 
to select $K$ to be open in $X$. The next lemma establishes that if $X$ is 0-dimensional, then one can find a compact open subset $K$ of $X$ homeomorphic to $\mathcal{C}_{a}$ for some $a$.

Lemma 4.5. Let $X$ be an uncountable 0-dimensional compact metric space. Then $X$ contains an open-and-closed subset homeomorphic to $\mathcal{C}_{a}$ for some countable ordinal number $a$.

Proof. Let $\alpha$ be the least ordinal such that there exists an open-and-closed, nondispersed subset $Y$ of $X$ with $Y^{(a)}$ perfect [25, Theorem 4.7]. Let $Y$ be such a subspace of $X$. Then for each $y$ in $\operatorname{Ker}(Y)$ and each open-and-closed neighborhood $U$ of $y$ included in $Y$, it follows by the minimality of a that $U^{(\gamma)}$ is perfect if and only if $\gamma \geq a$. Thus, $\left[Y^{(\gamma)} \sim \operatorname{Ker}(Y)\right] \cap U$ is nonempty and $y \in \mathrm{Cl}\left[Y^{(\gamma)} \sim \operatorname{Ker}(Y)\right]$ for each $\gamma<a$. Since this is true for each $y \in \operatorname{Ker}(Y)$,

$$
\operatorname{Ker}(Y) \subset \mathrm{Cl}\left[Y^{(\gamma)} \sim \operatorname{Ker}(Y)\right]
$$

for each $\gamma<a$. Therefore, by Lemma 4.3, $Y$ is a $\mathcal{C}_{a^{-s p a c e}}$.

The second "uncomplemented $C(X)$-subalgebra of $C(X)$ " result is stated in the next theorem. In contrast to Theorem 4.2, this theorem is applicable to 0 . dimensional compact metric spaces with a finite nonempty perfect derivative such as $\mathcal{C}$, the free union $\mathcal{C}_{1}+\mathcal{C}_{2}$ of $\mathcal{C}_{1}$ and $\mathcal{C}_{2}[13$, p. 127], and $\Gamma(\Omega) \times \mathcal{C}$. However, Theorem 4.6 does not include Theorem 4.2, since it is easy to construct spaces which satisfy the hypothesis of Theorem 4.2 but not of Theorem 4.6 (e.g., the subset $\left(\mathcal{C}_{\omega \omega} \times\{0\}\right) \cup\left(\mathcal{C}_{\times}[0,1]\right)$ of the unit square $)$.

Theorem 4.6. If a topological space $X$ contains an open, 0-dimensional compact metric subspace $K$ with $K^{(\omega)} \neq \varnothing$, then there is a map $\phi$ of $X$ onto itself such that $\phi^{0}[C(X)]$ is uncomplemented in $C(X)$.

Proof. If $K^{(n)}$ contains an isolated point for each positive integer $n$, this result follows by Theorem 4.2. Therefore, we may suppose $K^{(n)}$ is nonempty and perfect for some $n$ By Lemma 4.5, there is an open-and-closed subset $Y$ of $K$ homeomorphic to $C_{t}$ for some integer $t$. Let $S=\operatorname{Ker}(Y)$. By Lemma 3.5 there is a decomposition $D$ of $X$ with each plural set of $D$ a subset of $S$ such that $C(X / D)$ is uncomplemented in $C(X)$.

Let $q$ be the quotient map of $D$. We show $q(Y)$ is 0 -dimensional by examining the construction of $D$ in the proofs of Lemma 3.3 and Lemma 3.5. The notation used in these constructions will be preserved. Let $y \in Y$ and suppose $V$ is a neighborhood of $q(y)$ in $q(Y)$. If $y=x$ where $x$ is the point selected in the proof of Lemma 3.5, then there exists $n$ such that ${ }_{n}$ is included in the neighborhood $q^{-1}(V)$ of $x$. Then $q\left(O_{n}\right)$ is an open-and-closed neighborhood of $q(y)$ included in $V$. 
Next, suppose $y$ belongs to the complement of the closed set $F=\left(\bigcup_{n=1}^{\infty} E_{n}\right)$ $U\{x\}$ where $E_{n}$ and $x$ are defined in the proof of Lemma 3.5. Then there is an open-and-closed neighborhood $W$ of $y$ contained in $q^{-1}(V)$ which does not intersect $F$. In this case, $q(W)$ is an open-and-closed subset of $V$ in $q(Y)$ containing $q(y)$.

Finally, we suppose $y$ belongs to $E_{n}$ for some $n$ and restrict our attention to the selection of $M_{n}$ made in the proof of Lemma 3.3. This $M_{n}$ was constructed so that each plural set of $M_{n}$ would be a subset of $S_{n}=S \cap$ Int $\left(E_{n}\right)$. Suppose $q(y)$ belongs to one of the disjoint families $C_{m}$ selected in the proof of Lemma 3.3. Then $q(y)=\left\{a_{1}, a_{2}, \cdots, a_{z}\right\}$ where $z=1$ if $m=1$ and $z=k$ if $m>1$. We may assume that each set in the monotone neighborhood basis $\left\{V_{i j}\right\}$ of each $a_{i}$ selected in that proof is open-and-closed. Recall the sets $W_{i j}$ were selected so that $W_{i j} \subset V_{i j} \sim V_{i(j+1)}$. Since $q^{-1}(V)$ is a neighborhood of $q(y)$, there is a positive integer $p$ such that $V_{i p} \subset q^{-1}(V)$ for $i=1,2, \cdots, z$. If $W=$ $\bigcup_{i=1}^{z} V_{i(p k+1)}$, it is obvious from the construction of $M_{n}$ in Lemma 3.3 that $q(W)$ is an open-and-closed neighborhood of $q(y)$ contained in $V$.

On the other hand, suppose $q(y)$ is not a set in any $C_{i}$. Then, by the construction of $M_{n^{\prime}} q(y)$ is a nonlimit singleton set in $D$ and there is an open-andclosed neighborhood $W$ of $y$ included in $q^{-1}(V)$ which does not intersect any plural set of $D$. In this case, $q(W)$ is an open-and-closed neighborhood of $q(y)$ included in $V$. This completes the proof that $q(Y)$ is 0 -dimensional.

Since $Y$ is compact and $q$ is continuous, $q(Y)$ is compact. By a theorem of K. Morita and S. Hanai [19, Theorem 1] and also of A. H. Stone [26, Theorem 1], $q(Y)$ is metrizable. To establish $q(Y)$ is a $C_{t}$-space, it remains to be shown that $q(Y)^{(t)}$ is a nonempty perfect set and each point in $q(Y)^{(t)}$ is the limit of a wellordered sequence of points in $q(Y) \sim q(Y)^{(t)}$ homeomorphic to $\Gamma_{0}\left(\omega^{t}\right)$. Recall that each plural set in $D$ is a subset of $\operatorname{Ker}(Y)$. Consequently,

$$
\operatorname{Ker} q(Y)=q(\operatorname{Ker} Y)
$$

and

$$
q(Y) \sim \operatorname{Ker} q(Y)=q(Y \sim \operatorname{Ker} Y) .
$$

Since $\operatorname{Ker} q(Y)$ is perfect and the restriction of $q$ to $Y \sim \operatorname{Ker}(Y)$ is a homeomorphism, it follows from the fact that topological derivatives are preserved by homeomorphisms [23, Lemma $2.1(\mathrm{e})$ ] and equalities (1) and (2) above that

$$
\begin{aligned}
q(Y)^{(t)} & =[q(Y) \sim \operatorname{Ker} q(Y)]^{(t)} \cup[\operatorname{Ker} q(Y)] \\
& =q\left([Y \sim \operatorname{Ker} q(Y)]^{(t)}\right) \cup[\operatorname{Ker} q(Y)]=\operatorname{Ker} q(Y) .
\end{aligned}
$$

Thus, $q(Y)^{(t)}$ is perfect. 
Next, suppose $z$ is in $q(Y)^{(t)}$. By equality (1) above, there exists $y \in \operatorname{Ker}(Y)$ with $q(y)=z$. Let $\left\{x_{\mu}\right\}_{\mu<\omega t}$ be a well-ordered sequence in $Y \sim \operatorname{Ker}(Y)$ homeomorphic to $\Gamma_{0}\left(\omega^{t}\right)$ which converges to $z$. Since the restriction of $q$ to $Y \sim \operatorname{Ker}(Y)$ is a homeomorphism, it follows from line (2) that $\left\{q\left(y_{\mu}\right)\right\}_{\mu<\omega^{t}}$ is a well-ordered sequence in $q(Y) \sim \operatorname{Ker} q(Y)$ homeomorphic to $\Gamma_{0}\left(\omega^{t}\right)$ which converges to $z$. This completes the proof that $q(Y)$ is homeomorphic to $q(Y)$ (see Proposition 4.4). Since $Y$ is an open-and-closed set and $q$ is a homeomorphism of $X \sim Y$ onto $q(X) \sim q(Y), X$ is homeomorphic to $q(X)$.

Corollary 4.7. If $X$ is a 0-dimensional compact metric space with $X^{(\omega)} \neq \varnothing$, then there exists a map $\phi$ onto itself such that $\phi^{0}[C(X)]$ is an uncomplemented subalgebra of $C(X)$.

5. Applications of uncomplemented $C(X)$-subspaces of $C(X)$. The next two theorems are the uncomplemented analogues to Theorem 1 and Theorem 1 a, respectively, in [22] as they essentially replace "complemented" in the two theorems by A. Pel'czyński with "uncomplemented".

Theorem 5.1. Let $S$ be a compact metric space with $S^{(\omega)} \neq \varnothing$. If a Banach space $X$ contains a subspace $Y$ isomorphic to $C(S)$, then there is a subspace $Z$ of $Y$ such that $Z$ is isomorphic to $C(S)$ and $Z$ is not complemented in $X$.

Proof. Let $\mu$ be an isomorphism of $C(S)$ onto $Y$. First, suppose $S$ is countable. Then $S$ is dispersed, and, by Theorem 4.2, there is a map $\phi$ of $X$ onto itself such that $\phi^{0}[C(S)]$ is uncomplemented in $C(S)$. In this case, $\mu \phi^{0}[C(S)]$ is a subset of $Y$ isomorphic to $C(S)$ which is not complemented in $X$.

Next suppose $S$ is uncountable. By Milutin's Theorem (see [21, Theorem 8.5] or [11]) there is an isomorphism $\nu$ of $C(\mathcal{C})$ onto $C(S)$. By Corollary 4.7 there is a map $\phi$ of $\mathcal{C}$ onto itself such that $\phi^{0}[C(\mathcal{C})]$ is uncomplemented in $C(\mathcal{C})$. Then $\mu \nu \phi^{0}[C(\mathcal{C})]$ is an uncomplemented subspace of $X$ included in $Y$.

Theorem 5.2. Let $S$ be a 0-dimensional compact metric space with $S^{(\omega)} \neq \varnothing$. If a Banach space $X$ contains a subspace $Y$ (algebraically) isometrically isomorpbic to $C(S)$, then there is a subspace $Z$ of $Y$ such that $Z$ is (algebraically) isometrically isomorpbic to $C(S)$ and $Z$ is not complemented in $C(S)$.

Proof. Let $\mu$ be an (algebraic) isometric isomorphism of $C(S)$ onto $Y$. By Corollary 4.7 there is a map $\phi$ of $S$ onto itself such that $\phi^{0}[C(S)]$ is uncomplemented in $C(Y)$. Then $\mu \phi^{0}[C(S)]$ is an uncomplemented subspace of $C(X)$ contained in $Y$ which is (algebraically) isometrically isomorphic to $C(Y)$. 


\section{BIBLIOGRAPHY}

1. D. Amir, Continuous function spaces with the separable projection property, Bull. Res. Council of Israel Sect. F 10F (1962), 163-164. MR 27 \#566.

2. - Projections onto continuous function spaces, Proc. Amer. Math. Soc. 15 (1964), 396-402. MR $29 \# 2634$.

3. R. Arens, Projections on continuous function spaces, Duke Math. J. 32 (1965), 469-478. MR 31 \#6108.

4. J. W. Baker, Some uncomplemented subspaces of $C(X)$ of the type $C(Y)$, Studia Math. 36 (1970), 85-103. MR $43 \# 1113$.

19-27.

5. - Compact spaces hoemomorphic to a ray of ordinals, Fund. Math. 76 (1972),

6. - Dispersed images of bopological spaces and uncomplemented subspaces of $C(X)$, Proc. Amer. Math. Soc. 41 (1973), 309-314.

7. - Ordinal subspaces of topological spaces, General Topology and Appl. 3 (1973), 85-91.

8. - Projection constants for $C(S)$ spaces with the separable projection property, Proc. Amer. Math. Soc. 41 (1973), 201-204.

9. J. Baker and R. Lacher, Some mappings which do not admit an averaging operator (to appear).

10. N. Bourbaki, Eléments de mathématique. Part. 1. Les structures fondamentales de l'analyse. Livre III: Topologie générale, Actualités Sci. Indust., no. 1029, Hermann, Paris, 1947; English transl., Hermann, Paris; Addison-Wesley, Reading, Mass., 1966. MR 9, 261; 34 \#5044b.

11. S. Ditor, On a lemma of Milutin concerning averaging operators in continuous function spaces, Trans. Amer. Math. Soc. 149 (1970), 443-452.

12. - Averaging operators in $C(S)$ and lower semicontinuous sections of continuous maps, Trans. Amer. Math. Soc. 175 (1973), 195-208.

13. J. Dugundji, Topology, Allyn and Bacon, Boston, Mass., 1966. MR 33 \#1824.

14. N. Dunford and J. T. Schwartz, Linear operators. I. General theory, Pure and Appl. Math., vol. 7, Interscience, New York, 1958. MR $22 \# 8302$.

15. R. Engelking, Outline of general topology, PWN, Warsaw, 1965; English transl., North-Holland, Amsterdam; Interscience, New York, 1968. MR 36 \#4508; 37 \#5836.

16. F. Hausdorff, Set theory, 2nd ed., Chel sea, New York, 1957.

17. E. Hewitt and K. Stromberg, Real and abstract analysis. A modern treatment of the theory of functions of a real variable, Springer-Verlag, New York, 1965, MR 32 \#5826.

18. J. Kelley, General topology, Van Nostrand, Princeton, N. J., 1955. MR 16, 1136.

19. K. Morita and S. Hanai, Closed mappings and metric spaces, Proc. Japan Acad. 32 (1956), 10-14. MR 19, 299.

20. A. Pel'czyński and Z. Semadeni, Spaces of continuous functions. III. Spaces $C(\Omega)$ for $\mathbb{Q}$ without perfect subsets, Studia Math. 18 (1959), 211-222. MR 21 \$6528.

21. A. Pel czyński, Linear extensions, linear averagings, and their applications to linear topological classification of spaces of continuous functions, Rozprawy Mat. 58 (1968), 92 pp. MR 37 \#3335.

22. - $O_{n} C(S)$-subspaces of separable Banach spaces, Studia Math. 31 (1968), 513-522. MR $38 \# 2578$. 
23. R. S. Pierce, Existence and uniqueness theorems for extensions of zero-dimensional compact metric spaces, Trans. Amer. Math. Soc. 148 (1970), 1-21. MR 40 \#011.

24. Z. Semadeni, Sur les ensembles clairsemés, Rozprawy Mat. 19 (1959), 1-39. MR 21 \#571.

25. W. Sierpinski, General topology, 2nd ed., Univ. of Toronto Press, Toronto, 1956.

26. A. H. Stone, Metrizability of decomposition spaces, Proc. Amer. Math. Soc. 7 (1956), 690-700. MR 19, 299.

DEPARTMENT OF MATHEMATICS, FLORIDA STATE UNIVERSITY, TALLAHASSEE, FLORIDA 32306

Current address: Department of Mathematics, Kent State University, Kent, Ohio 44242 